\title{
Formation Mechanism of Local Thickness Profile of Silicon Epitaxial Film
}

Hitoshi Habuka*, Shin-ichi Fukaya, Aya Sawada, Takashi Takeuchi and Masahiko Aihara

Department of Chemical Engineering Science, Yokohama National University, 79-5 Tokiwadai, Hodogaya, Yokohama, Kanagawa 240-8501, Japan

* Phone and Fax: +81-45-339-3998, e-mail: habuka@chemeng.bsk.ynu.ac.jp

From the viewpoint to obtain a very flat semiconductor silicon epitaxial film, the influence of an epitaxial reactor design on a local thickness profile of a silicon epitaxial film is studied. The characteristic thickness valleys formed in a trichlorosilane-hydrogen system using a horizontal cold wall single-wafer epitaxial reactor at atmospheric pressure are concluded to have a quantitative relationship with the local decrease in the transport rate of trichlorosilane induced by the increase in the velocity, the increase in the temperature and the decrease in the trichlorosilane concentration in the gas phase, which are induced by the gas inlet design.

Keywords: silicon epitaxial growth, flatness, growth rate, transport phenomena PACS Codes: 81.05.Cy, 81.10.Bk, 81.15.Gh, 81.15.Aa 


\section{Introduction}

A silicon epitaxial wafer has been recognized to be suitable for manufacturing current and future advanced microelectronics devices. In the future, the further shrinking design rule for microelectronic devices will require a very high flatness and excellent nanotopography of the silicon epitaxial wafer $[1,2]$. In order to satisfy this increasing demand, many try-and-error approaches have been performed by modifying the reactor geometry, such as a multi-inlet in the horizontal single-wafer epitaxial reactor. Although the multi-inlet is empirically known to be able to adjust the entire trend of the epitaxial film thickness, such the reactor often locally shows a slight thickness variation [3]. In order to perform the systematic approach to optimize the reactor design and to minimize the local thickness variation, its formation mechanism has been studied using the theoretical calculation $[4,5]$, accounting for the transport phenomena and the exact geometry of the epitaxial reactor including the multi-inlet. Although these studies could successfully reproduce the trend of the epitaxial growth rate distribution, its quantitative relationship among many complicated factors in the reactor has not been extracted and clarified. The simple relationship is necessary for developing the practical way to design the reactor. Additionally, this information will help the scientists and engineers using a chemical vapor deposition reactor with surveying the optimum condition for film formation.

Therefore, in this study which advances our previous work [5], the trichlorosilane transport rate is especially focused on, among various factors for the chemical vapor deposition, in order to clarify the dominant formation mechanism of the local growth rate distribution and the local thickness profile of the silicon epitaxial film in the industrially used horizontal cold wall single-wafer epitaxial reactor. The local thickness valley will be shown to be simply formed by the local gas phase temperature distribution. 


\section{Theoretical Methods}

In this section, the reactor geometry and the theoretical method in this study are briefly described.

Figure 1 shows the geometry of the industrially-used horizontal cold wall single-wafer reactor used for silicon epitaxial film growth. A gas mixture of trichlorosilane and hydrogen is introduced at atmospheric pressure through the multi-inlet into the reactor and reacts on the surface of a $200-\mathrm{mm}$-diameter silicon substrate which is held horizontally and rotates at $20 \mathrm{rpm}$. The multi-inlet has three sections, left (L), center (C) and right $(\mathrm{R})$, which are divided using inlet plates set at the center of the inlet and on the right and left. The gas mixture flows between the inlet plates, and it climbs up a stair to approach the susceptor and the substrate. The average gas velocity in the multi-inlet is $0.21 \mathrm{~m} / \mathrm{s}$ for the $\mathrm{L}$ and $\mathrm{R}$ sections, $0.78 \mathrm{~m} / \mathrm{s}$ for the $\mathrm{C}$ section, at the atmospheric pressure of $1.0133 \times 10^{5} \mathrm{~Pa}$. At the inlet of the reactor, the concentration of trichlorosilane $\left(\mathrm{SiHCl}_{3}\right)$ in an ambient hydrogen $\left(\mathrm{H}_{2}\right)$ is $3.5 \%$. The silicon substrate temperature is set at $1400 \mathrm{~K}$; the temperature of the quartz walls near the silicon substrate is $750 \mathrm{~K}$, like a cold wall reactor used in our previous studies [6, $7]$.

In order to calculate the epitaxial growth rate, the transport and epitaxy model [6, 7] is applied to this study. This model consists of theoretical equations for the transport phenomena of the mass, momentum, energy and chemical species and the surface reactions to form the silicon epitaxial film. The discretized equations are coupled and solved using the SIMPLE algorithm [8] on a CFD software package, Fluent version 5.5 (Fluent, Inc.). The physical properties used in this study are the same as those in our 
previous studies $[6,7]$.

The key processes for the silicon epitaxial film growth are represented by the following two chemical reactions:

Chemisorption of $\mathrm{SiHCl}_{3}$ :

$$
\mathrm{SiHCl}_{3} \rightarrow * \mathrm{SiCl}_{2}+\mathrm{HCl}
$$

Decomposition of chemisorbed species, ${ }^{*} \mathrm{SiCl}_{2}$ (production of $\mathrm{Si}$ film):

$$
* \mathrm{SiCl}_{2}+\mathrm{H}_{2} \rightarrow \mathrm{Si}+\mathrm{HCl}
$$

In these equations, the chemical species chemisorbed on the silicon surface are indicated using '*'. The growth rate of silicon epitaxial film, $V \quad\left(\mathrm{~mol} \mathrm{~m} \mathrm{~m}^{-2} \mathrm{~s}^{-1}\right)$, is described as follows:

$$
V=\frac{k_{\mathrm{ad}} k_{\mathrm{r}}\left[\mathrm{SiHCl}_{3}\right]\left[\mathrm{H}_{2}\right]}{k_{\mathrm{ad}}\left[\mathrm{SiHCl}_{3}\right]+k_{\mathrm{r}}\left[\mathrm{H}_{2}\right]}
$$

$[i]$ is the mole concentration of species $i\left(\mathrm{~mol} \mathrm{~m}^{-3}\right)$ at the silicon substrate surface, $k_{\mathrm{ad}}$ is the chemiforption rate constant $\left(\mathrm{m} \mathrm{s}^{-1}\right)$, and $k_{\mathrm{r}}$ is the decomposition rate constant ( $\mathrm{m}$ $\left.\mathrm{s}^{-1}\right)$. The rate constants, $k_{\mathrm{ad}}$ and $k_{\mathrm{r}}$, have been reported in our previous studies $[6,7]$.

\section{$\underline{\text { Results and Discussion }}$}

After briefly summarizing the results in our previous study [5], the formation mechanism of the local thickness valley is discussed.

\section{Local thickness valley}

Fig. 2(a) shows the contour lines of the calculated silicon epitaxial growth rate distribution normalized using the growth rate at the center of the substrate. Fig. 2(b) shows the contour lines of the calculated gas phase temperature at $0.003 \mathrm{~m}$ above the silicon substrate. Slanted squares, followed by broken lines, indicate the position of the three inlet plates. The low growth rate regions along the broken lines in Fig. 2(a) have 
the higher gas velocity at its front edge, the higher temperature in the gas phase, as shown using letter $\mathrm{H}$ in Fig. 2(b), and thus the lower trichlorosilane concentration than the other regions. At the front edge of the substrate, the variation of the epitaxial growth rate in the traverse direction is estimated to be $2-3 \%$.

The details of the entire transport phenomena and the growth rate have been written in our previous study [5].

\section{Transport rate of trichlorosilane}

In this section, a formation mechanism of the local growth rate distribution is discussed.

In our previous study [5], the reason to form the characteristic local distribution of the epitaxial growth rate was qualitatively assumed as follows: (1) The plate of the multi-inlet generates the region with a small gas velocity around itself due to the effect of gas viscosity. (2) In this slow stream region, the gases are rapidly heated by the hot substrate and susceptor; the gas volume is expanded to decrease the trichlorosilane gas concentration (per unit volume); and the diffusivity of trichlorosilane gas simultaneously increases. (3) The growth rate decrease due to the decrease in the trichlorosilane gas concentration cannot be compensated by its increase due to the increase in the trichlorosilane gas diffusivity. However, a quantitative description of this assumption has not been evaluated.

Therefore, the transport rate of trichlorosilane is focused on. Taking into account the transport limited region with a suppressed gas phase reaction in the industrial silicon epitaxial growth $/$ condition, the silicon epitaxial film growth rate is considered to be dominated by the transport rate of the source gas from the gas phase to the substrate, $R_{\text {Trans }}\left(\mathrm{kg} \mathrm{m}^{-2} \mathrm{~s}^{-1}\right)$. The behavior of $R_{\text {Trans }}$ can be evaluated assuming the diffusion transport of the source gas species from the gas phase to the substrate surface, 
as expressed in equation (4), using the variables shown in Fig. 3.

$$
R_{\text {Trans }}=D\left(T_{\text {ave }}\right) \frac{C_{\text {gas }}\left(T_{\text {gas }}\right)-C_{\text {surface }}}{h}+D^{\mathrm{T}} \frac{1}{T_{\text {ave }}} \frac{T_{\text {gas }}-T_{\text {surface }}}{h} \text {, }
$$

where $T_{\text {ave }}(\mathrm{K})$ is the average temperature in the layer for the diffusion transport of the trichlorosilane gas.

$$
T_{\text {ave }}=\frac{T_{\text {gas }}+T_{\text {surface }}}{2}
$$

$T_{\text {surface }}(\mathrm{K})$ and $T_{\text {gas }}(\mathrm{K})$ are the temperatures of the substrate surface and the representative temperature in the gas phase, respectively. $D\left(T_{\text {ave }}\right)\left(\mathrm{m}^{2} \mathrm{~s}^{-1}\right)$ is the diffusivity of the trichlorosilane gas evaluated at $T_{\text {ave }} C_{\text {gas }}\left(T_{\text {gas }}\right)\left(\mathrm{kg} \mathrm{m}^{-3}\right)$ is the concentration of the trichlorosilane gas in the gas phase which has the temperature of $T_{\text {gas. }} C_{\text {surface }}\left(\mathrm{kg} \mathrm{m}^{-3}\right)$ is the concentration of the trichlorosilane gas at the substrate surface. $h(\mathrm{~m})$ is the distance for the diffusion transport from the gas phase to the substrate surface. $C_{\mathrm{gas}}\left(T_{\mathrm{gas}}\right)$ is obtained as follows:

$$
C_{\text {gas }}\left(T_{\text {ave }}\right)=C_{0} \frac{300}{T_{\text {gas }}}
$$

$C_{0}\left(\mathrm{~kg} \mathrm{~m}^{-3}\right)$ is the concentration of trichlorosilane gas at the inlet of the reactor. In this study, $C_{0}$ is $0.22 \mathrm{~kg} \mathrm{~m}^{-3}$, which corresponds to the mole fraction of the trichlorosilane gas in hydrogen gas of $0.035 . C_{\text {surface }}$ is assumed to be zero, since the trichlorosilane gas concentration at the substrate surface at high temperatures in the transport limited region is significantly smaller than $C_{\mathrm{gas}}\left(T_{\mathrm{gas}}\right)[6,7,9] . h$ is $0.01 \mathrm{~m} . T_{\text {surface }}$ is fixed at $1400 \mathrm{~K}$. Although the thermal diffusion is known to influence the species transport in the cold-wall reactor [9], it simply decreases the diffusion transport of the source gas to the substrate surface by several percent without changing its entire trend. Therefore, this study ignores the thermal diffusion term in equation (4). The binary diffusion 
coefficient of trichlorosilane in hydrogen is estimated by the method described in the literature [10], and is expressed as a function of temperature.

The influence of various factors in the trichlorosilane transport in the gas phase is discussed using equations (4)-(6). The concentration of trichlorosilane gas in the gas phase and its diffusivity in the hydrogen gas are calculated and shown in Fig. 4 as a function of $T_{\text {gas. }} D\left(T_{\text {ave }}\right)$ in the cold-wall reactor shows a moderate increase, since $T_{\text {ave }}$ moderately increases because of fixed $T_{\text {surface }}$ in equations (5). In contrast to the change in $D\left(T_{\mathrm{ave}}\right)$, the decrease in $C_{\mathrm{gas}}\left(T_{\mathrm{gas}}\right)$ due to the volume expansion is significant. As shown in Fig. 4 , the values of $D\left(T_{\text {ave }}\right)$ and $C_{\text {gas }}\left(T_{\text {gas }}\right)$ at $T_{\text {gas }}$ of $1400 \mathrm{~K}$ become $200 \%$ and $25 \%$ of those at room temperature, respectively. As a result, $R_{\text {Trans }}$ in the cold-wall reactor decreases with increasing $T_{\text {gas, }}$ as shown in Fig. 5 .

Next, the value of the growth rate variation is further studied. Fig. 6 shows the calculated transport rate variation of trichlorosilane induced by the local $T_{\text {gas }}$ difference. Here, $T_{\text {surface }}$ and the local $T_{\text {gas }}$ difference are fixed to be $1400 \mathrm{~K}$ and $100 \mathrm{~K}$, respectively. Since the $R_{\text {Trans }}$ decreases with increasing $T_{\text {gas; }}$ its slope simultaneously decreases, as shown in Fig. 5, thus the transport rate variation induced by the local $T_{\text {gas }}$ difference decreases, as shown in Fig. 6. As shown in Fig. 2(b), the front edge of the substrate has temperature difference of $60-80 \mathrm{~K}$ between the center high temperature region (indicated using letter A) and its right and left regions (indicated using letter B) having low temperatures. This temperature difference at $0.003 \mathrm{~m}$ above the substrate corresponds to the local $T_{\text {gas }}$ difference larger than $100 \mathrm{~K}$ at $h$ of $0.01 \mathrm{~m}$. The transport rate variation for $100 \mathrm{~K}$ is calculated to be $2-3 \%$ in the $T_{\text {gas }}$ range of $1000-1200 \mathrm{~K}$ in Fig. 6. This coincides with the value of the growth rate variation at the front edge of the substrate between the regions A and B in Fig. 2(a), and also agrees with the value of the thickness variation reported in our previous study [5]. Therefore, the non-uniform temperature distribution in the traverse direction in the gas phase is 
concluded to result in the local variation of the epitaxial growth rate. Additionally, this induces the locally non-uniform silicon epitaxial film thickness distribution, because this symmetrically formed growth rate distribution in the traverse direction of the horizontal single-wafer cold-wall reactor, in Fig. 2(a), cannot be compensated or flattened by the function of the substrate rotation [11], taking an average of the epitaxial growth rate along the concentric circle.

\section{Conclusions}

In order to clarify the formation mechanism of the local silicon epitaxial film thickness variation in a trichlorosilane-hydrogen system, the influence of the locally non-uniform transport rate distribution of trichlorosilane gas in the gas phase above a substrate on a silicon epitaxial film growth is studied. Since the significant decrease in the trichlorosilane gas concentration cannot be sufficiently compensated by the moderate increase in its diffusivity, due to the local gas temperature increase in the horizontal cold wall reactor, the epitaxial growth rate and the thickness become locally non-uniform. The inlet design should be performed taking into account its influence on the gas phase temperature distribution as same as the adjustability of the entire epitaxial film thickness profile. 


\section{$\underline{\text { References }}$}

[1] C. S. Xu, E. Zhao, R. Jairath and W. Krussel, Electrochem. Solid-State Lett., 1 (1998) 181.

[2] T. Fukuda, Denshizairyo, p. 89 (Feb. 2001). [in Japanese]

[3] Y. Ohta, MOS Device Epitaxial Wafers, eds: H. Tsuya, H. Shimizu and H. Yamamoto, (Realize, Inc., Tokyo, 1998) p. 59. [in Japanese]

[4] H. Habuka, Jpn. J. Appl. Phys., 40 (2001) 6041.

[5] H. Habuka, S. Fukaya, A. Sawada, T. Takeuchi and M. Aihara, Jpn. J. Appl. Phys., 41 (2002) 5692.

[6] H. Habuka, T. Nagoya, M. Mayusumi, M. Katayama, M. Shimada and K. Okuyama, J. Crystal Growth, 169 (1996) 61.

[7] H. Habuka, M. Katayama, M. Shimada and K. Okuyama, J. Crystal Growth, 182 (1997) 352.

[8] S. V. Patankar, Numerical Heat Transfer and Fluid Flow, (McGraw-Hill, New York, 1980).

[9] H. Habuka, M. Katayama, M. Shimada and K. Okuyama, Jpn. J. Appl. Phys., 33 (1994) 1977.

[10] R. C. Reid, J. M. Prausnitz and B. E. Poling, The Properties of Gases and Liquids (McGraw-Hill, New York, 1987) 4th ed.

[11] H. Habuka, T. Nagoya, M. Katayama, M. Shimada and K. Okuyama, J. Electrochem. Soc., 142 (1995) 4272. 


\section{Figure captions}

Fig. 1 Industrially-used horizontal cold wall single-wafer reactor.

Fig. 2 (a) Contour lines of the calculated growth rate of silicon epitaxial film normalized using the growth rate at the center of the substrate. (b) Contour lines of the calculated gas temperature $(K)$ at $0.003 \mathrm{~m}$ above the silicon substrate and its central lateral cross section. Slanted squares indicate the position of the three inlet plates. Broken lines with arrows indicate the position of the downstream region corresponding to the inlet plate. Letter $\mathrm{A}$ indicates the center region along the broken line, letter $B$ indicates the intermediate region between the broken lines.

Fig. 3 Definition of the variables in this study.

Fig. 4 Changes in the diffusivity, $D\left(T_{\text {ave }}\right)$, and the concentration, $C_{\text {gas }}\left(T_{\text {gas }}\right)$, of the trichlorosilane gas in the hydrogen gas with the temperature in the gas phase, $T_{\text {gas, }}$ in the cold-wall reactor. The mole fraction of the trichlorosilane gas in the hydrogen gas is 0.035 .

Fig. 5 Changes in the transport rate of the trichlorosilane gas to the substrate surface with the temperature in the gas phase, $T_{\mathrm{gas}}$, in the cold-wall reactor, using equation (4). $T_{\text {surface }}$ is fixed at $1400 \mathrm{~K}$.

Fig. 6 Calculated transport rate variation of trichlorosilane gas induced by the local $T_{\text {gas }}$ difference of $100 \mathrm{~K} . T_{\text {surface }}$ is fixed at $1400 \mathrm{~K}$. 

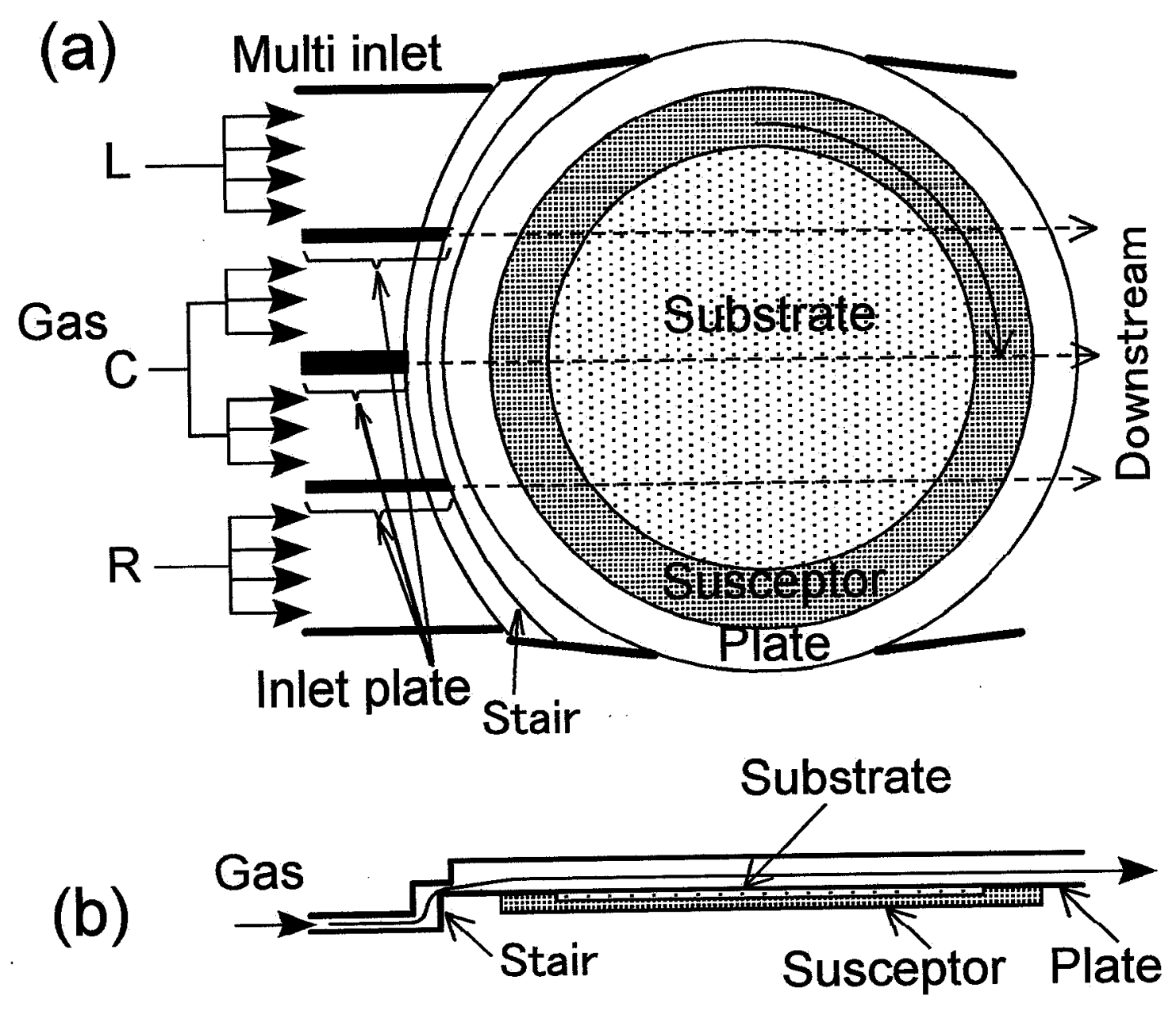

Fig. 1 

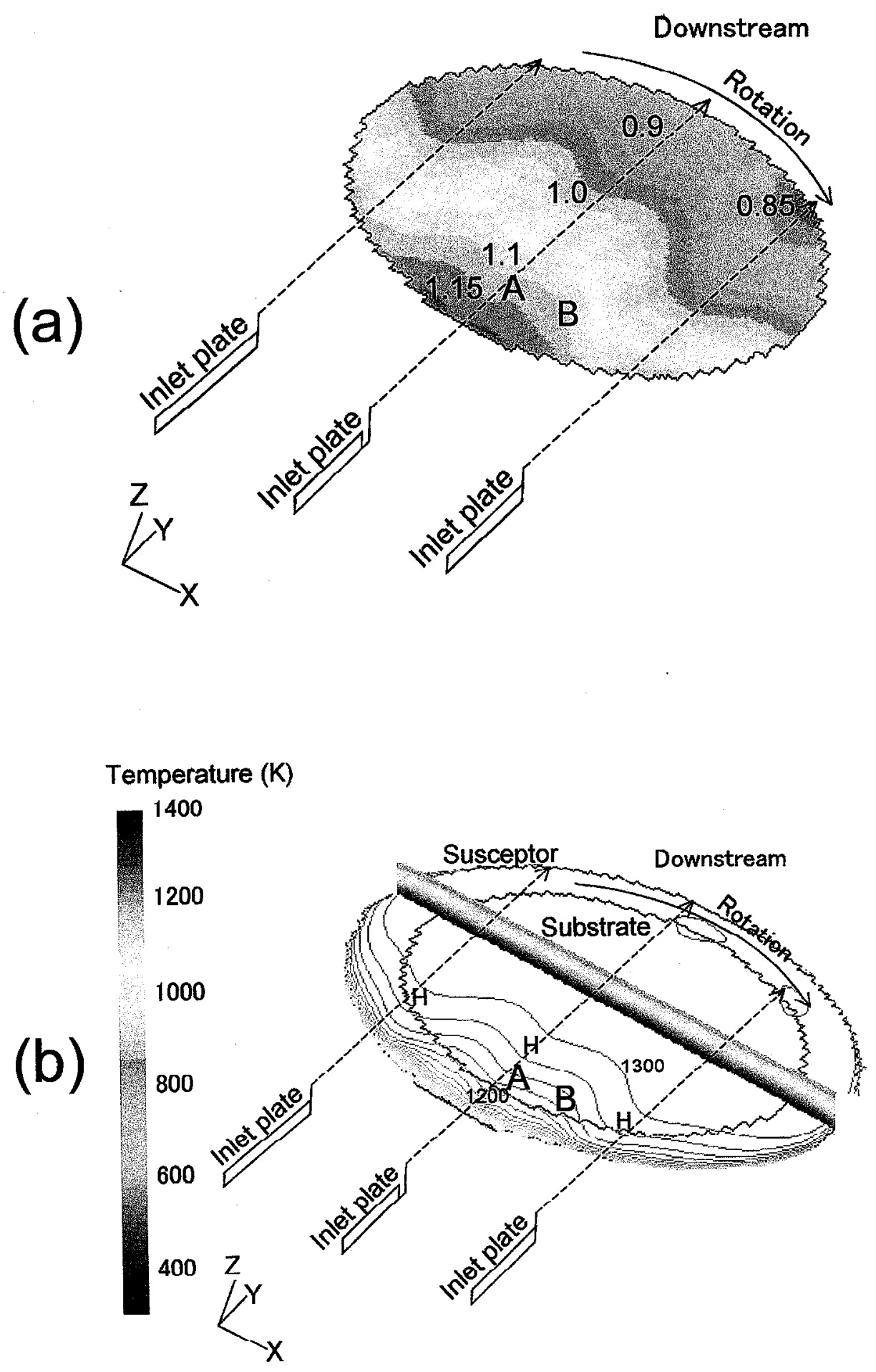

Fig. 2 


\section{Top wall}

\section{Gas phase}

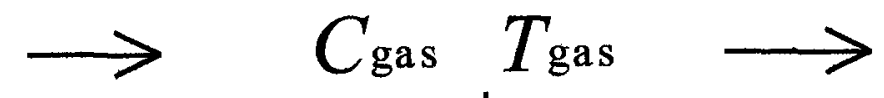

\begin{tabular}{r|l} 
Height, $h$ & Diffus ivity, $D\left(T_{\text {ave }}\right)$ \\
$C_{\text {surface }}$ & $\downarrow T_{\text {surface }}$
\end{tabular}

Substrate

$$
T_{\mathrm{ave}}=\frac{T_{\mathrm{gas}}+T_{\text {surface }}}{2}
$$

Fig. 3 


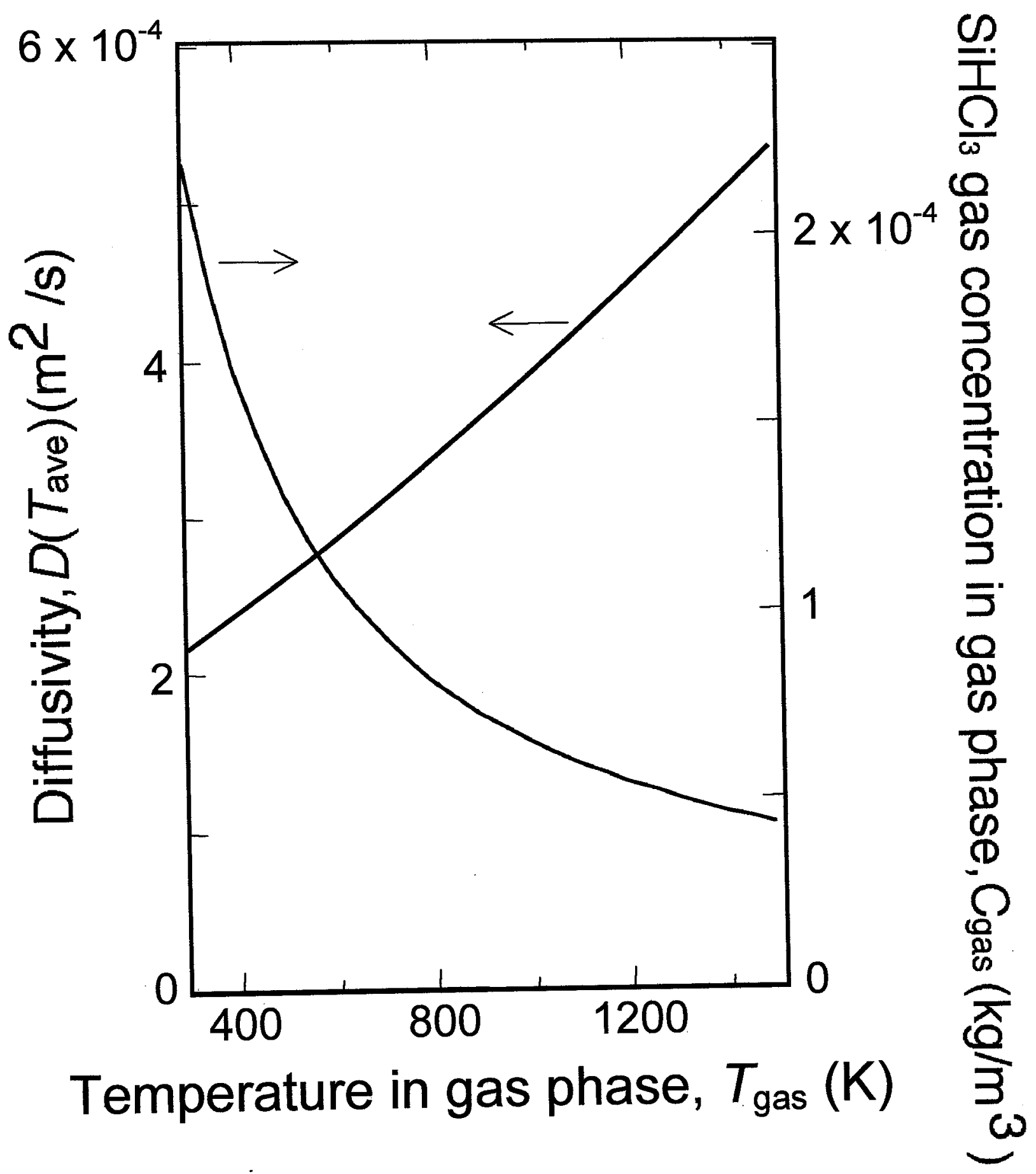

Fig. 4 


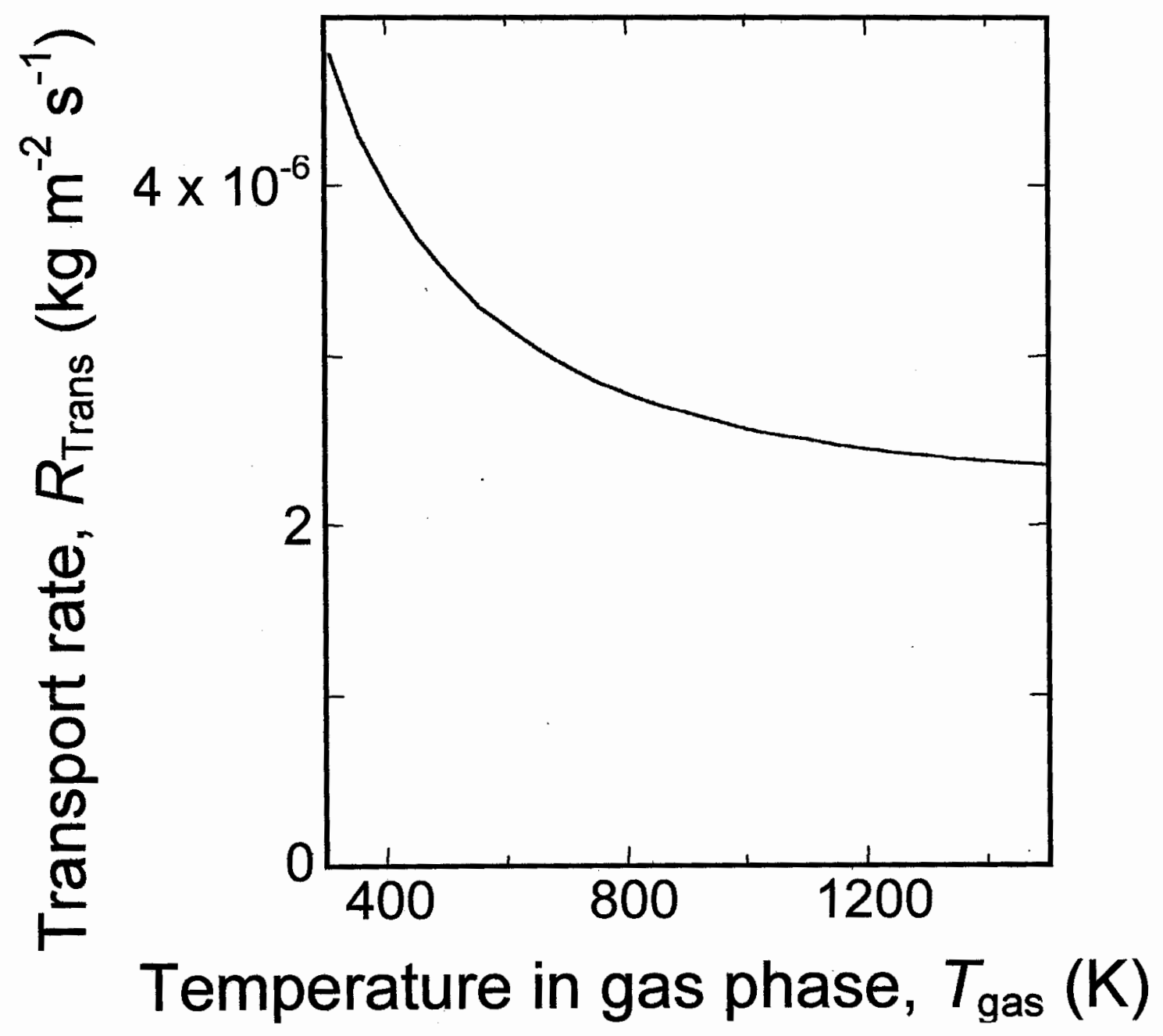

Fig. 5 


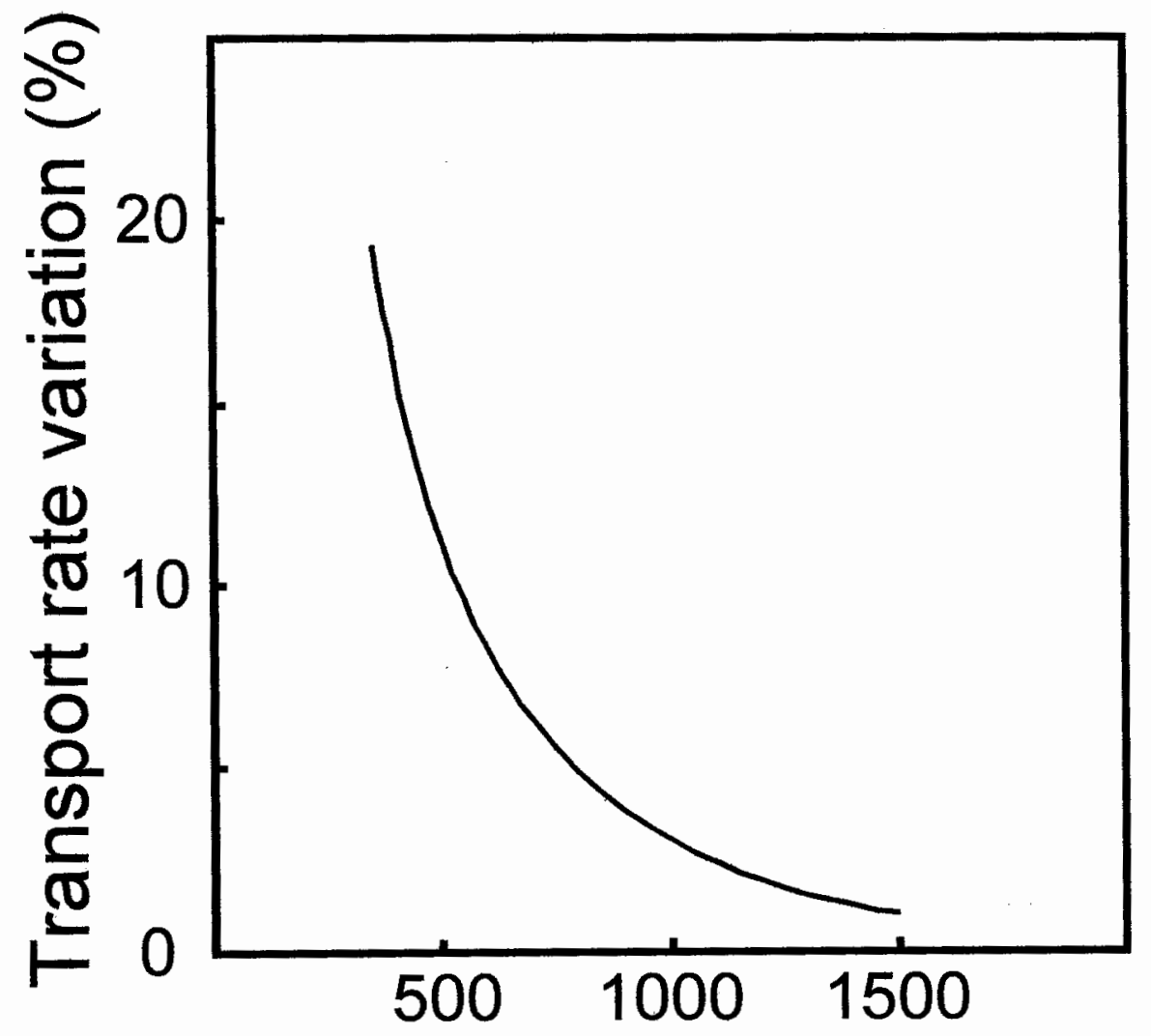

Temperature in gas phase, $T_{\text {gas }}(\mathrm{K})$

Fig. 6 